\title{
Molecular Delineation of De Novo Small Supernumerary Marker Chromosomes in Prenatal Diagnosis, A Retrospective Study
}

\section{Shuang Hu}

First Affiliated Hospital of Zhengzhou University

Xiangdong Kong ( $\square$ kongxd@263.net )

First Affiliated Hospital of Zhengzhou University

\section{Research Article}

Keywords: amniotic fluid, small supernumerary marker chromosome, SNP array, CNV-seq, prenatal diagnosis

Posted Date: December 8th, 2021

DOI: https://doi.org/10.21203/rs.3.rs-927187/v2

License: (c) (i) This work is licensed under a Creative Commons Attribution 4.0 International License. Read Full License 


\section{Abstract}

\section{Background}

To define the genotype-phenotype correlation of small supernumerary marker chromosomes (sSMCs) and conduct precise genetic counseling, we retrospectively searched and reviewed de novo SSMC cases detected during prenatal diagnosis at The First Affiliated Hospital of Zhengzhou University. Chromosome karyotypes of 20,314 cases of amniotic fluid from pregnant women were performed. For 16 samples with de novo sSMCs, 10 were subjected to single-nucleotide polymorphism (SNP) array or low-coverage massively parallel copy number variation sequencing (CNV-seq) analysis.

\section{Results}

Among the 10 sSMC cases, two sSMCs derived from chromosome 9, and three sSMCs derived from chromosomes 12, 18 and 22. The remaining 5 cases were not identified by SNP array or CNV-seq because they lacked euchromatin or had a low proportion of mosaicism. Four of them with a karyotype of $47, \mathrm{XN},+$ mar presented normal molecular cytogenetic results (seq[hg19] 46,XN), and the remaining patient with a karyotype of 46,XN,+mar presented with Turner syndrome (seq[hg19] 45,X). Five sSMC samples were mosaics of all 16 cases.

\section{Conclusion}

Considering the variable origins of SSMCs, further genetic testing of SSMCs should be performed by SNP array or CNV-seq. Detailed molecular characterization would allow precise genetic counseling for prenatal diagnosis.

\section{Background}

Small supernumerary marker chromosomes (sSMCs) are abnormal chromosome fragments smaller than chromosome 20 in size that have been reported to occur in approximately $0.043 \%$ of live births and $0.075 \%$ of prenatal cases $[1,2]$. Small supernumerary marker chromosomes can be identified but not characterized by routine G-banding karyotypes alone. sSMCs can derive from any chromosome and sometimes present with mosaic conditions and have morphologies such as isodicentric bisatellited, ring, isodicentric or centric minute, and inverted duplicated shapes [3]. A previous study showed that $70 \%$ of sSMCs are de novo and that $30 \%$ are inherited either from mothers (20\%) or fathers (10\%) [4]. Most sSMCs derive from the short arms and pericentromeric regions of acrocentric chromosomes; a small fraction originate from nonacrocentric autosomes [5]. Owing to the diverse origins of chromosomes and various ratios of mosaicism or other concomitant chromosome imbalances, the phenotype of patients with sSMC varies significantly from normal to serious illness, including intellectual disability, craniofacial abnormalities, autistic behaviors and growth retardation[6].

sSMC carrying specific genomic content are associated with defined clinical syndromes [7-10]. For example, isochromosome 12p is associated with Pallister-Killian syndrome (OMIM 601803), sSMC(15) 
and Prader-Willi Syndrome (OMIM176270)/Angelman Syndrome (OMIM105830)(PWS/AS) (in the case of UPD or an in-parallel deletion of a PWS/AS critical region) isochromosome 18p syndrome. sSMCs derived from chr22 are associated with cat eye syndrome CES (OMIM115470) when the cat eye critical region (CES-CR) is present twice on the SSMC. However, most SSMC carriers present normal development; thus, the complexity of the phenotype-genotype correlation makes prenatal diagnosis challenging. Thus, molecular delineation of sSMCs is very meaningful for genetic counseling in the clinic.

With the development of science and technology, an increasing number of molecular cytogenetic techniques are being analyzed to characterize SSMCs, including fluorescence in situ hybridization (FISH), single-nucleotide polymorphism (SNP) array, comparative genomic hybridization (CGH) and highthroughput sequencing for copy number variation (CNV-seq).

In the present study, we retrospectively collected 16 sSMC karyotype cases from 20,413 pregnant women by amniocentesis from our prenatal and diagnosis center from January 2017 to January 2020. Molecular cytogenetic characterization involved analysis by conventional G-banding and SNP array or CNV-seq, and then appropriate genotype-phenotype correlations for prenatal genetic counseling were provided by our genetic counselors. The primary aim of our research was to provide a genetic-based prenatal diagnosis of sSMC in China.

This study was approved by the Medical Ethics Committee of the First Affiliated Hospital of Zhengzhou University. All analyzed samples were obtained after obtaining signed informed consent.

\section{Materials And Methods}

\section{Subjects}

Our study recruited 20,314 pregnant women with the need for prenatal cytogenetic diagnosis who visited the clinic of the prenatal diagnosis center of the First Affiliated Hospital of Zhengzhou University between January 2017 and January 2020. The indications for prenatal diagnosis included a high-risk pregnancy by NIPT, a high risk of trisomy for chromosomes $21 / 18 / 13$ by serum screening, abnormal ultrasound, advanced maternal age (age $\geq 35$ ), and a history of abnormal pregnancy. Conventional G-banding karyotyping was performed for every amniotic fluid sample. SSMC was detected in 16 pregnancies. The women were at 17-24 weeks gestation when undergoing amniocentesis. Abnormal cell lines with sSMCs were detected in two independent cultures.

\section{Methods}

\section{Karyotyping analysis}

We retrospectively searched and reviewed SSMC cases detected during prenatal diagnosis in The First Affiliated Hospital of Zhengzhou University. Amniotic fluid specimens $(10 \mathrm{ml})$ were collected by amniocentesis under the guidance of ultrasonography and then centrifuged at $1500 \mathrm{r} / \mathrm{min}$ for 8 minutes. The supernatant was discarded, and the remaining liquid was mixed and incubated with two $5 \mathrm{ml}$ aliquots 
of amniocyte culture medium (Gibco, USA and Israel). Cells were cultured in an incubator at $37^{\circ} \mathrm{C}$ and $5 \%$ CO2 for approximately 9 days. When colonies reached more than 15 , the cells were collected followed by sections, and classical G-banding karyotyping was performed for every sample. The banded sections were scanned using a Zeiss Automated Nuclear Scanning System. For each sample, 5 mitotic figures were analyzed with 30 counts (abnormal karyotype with 100 counts). For these abnormal karyotypes, secondline cells were also managed as the first line.

\section{SNP microarray.}

SNP-array analysis was conducted using the Affymetrix CytoScan platform (Affymetrix, Santa Clara, CA, USA) according to the manufacturer's protocol for a whole-genome scan of amniotic cell DNA for Cases 15 and 16. Molecular karyotype analysis was conducted using KaryoStudio version 1.4.3.0 (Illumina, San Diego, CA).

\section{CNV-seq}

Low-coverage massively parallel copy number variation sequencing (CNV-seq) was conducted with the Illumina platform for patient samples of Cases 7-14. A total of $10 \mathrm{ng}$ of input DNA is sufficient for accurate CNV-seq diagnosis, though $50 \mathrm{ng}$ is optimal. Genomic DNA (10 ng) was fragmented, and a DNA library was constructed as previously described [7]. Multiple libraries were indexed and pooled into a single lane and sequenced with a NextSeq CN500 instrument (Illumina, Inc.) to produce approximately 5 million single-end reads of $45 \mathrm{bp}$ (including the $8 \mathrm{bp}$ index sequence). Genomic Variants Database (http://projects.tcag.ca/variation), DECIPHER (http://decipher.sanger.ac.uk/), ClinVar (https://www.ncbi.nlm.nih.gov/clinvar/), OMIM (http://www.ncbi.nlm.nih.gov/omim) and ClinGen (https://www.clinicalgenome.org/) were used for interpretation and classification of the clinical significance of candidate CNVs according to previously reported guidelines [11, 12].

\section{Results}

\section{The whole distribution of SSMC and mosaicism}

In our survey, 16 de novo sSMC cases were detected in 20,314 amniotic fluid samples from pregnant women by conventional G-banding karyotyping; among the 16 sSMCs, 5 cases exhibited mosaicism. Eleven of the 16 samples with de novo sSMCs were successfully subjected to single-nucleotide polymorphism (SNP) array or low-coverage massively parallel copy number variation sequencing (CNVseq) analysis (Cases 7-16). The remaining 6 (Cases 1-6) were not characterized further.

Of the 10 sSMCs, only 5 were endowed with detectable euchromatin. For the remaining 5 sSMCs, their CNV-seq results were negative and did not show any pathogenic aberrations. Of these, the CNV-seq results for four were seq[hy19] 46,XN (Cases $8,9,10,11$ ). One sSMC with a karyotype of $46, X,+$ mar was a case of Turner syndrome with a CNV-seq result of $45, X$ (Case 7 ). 
The remaining 5 sSMCs with chromosome loss or gain derived from chromosome 12, chromosome 18, and chromosome 22. The remaining two sSMCs originated from chromosome 9.

Cytogenetic analysis displayed mosaic sSMCs in 5 of 16 cases (Cases 1,3,10,12 and 15). Comprehensive analysis of the two cell lines revealed that their mosaic level ranged from $4 \%$ to $74 \%$.

\section{Chromosome origin and genotype-phenotype analysis}

For Cases 1 to 6, only G-banding karyotyping was done. For Cases 7 to 11, CNV-seq analysis revealed that no genes were involved in the sSMCs. In Cases 12 to 16 , the sSMCs contained significant chromosome fragments (Table 1). The following delineates the correlation between genotype and phenotype in detail.

For Case 12, the sSMC derived from chromosome 9, with a gain of approximately $10 \mathrm{Mb}$ of the 9p12p21 region (seq[hg19] 9p12p21(28600000-38780000)×3囚40\%区) (Figure 1-A2). Karyotype analysis showed approximately $70 \%$ of analyzed metaphases with the SSMC; thus, the karyotype was defined as $47, \mathrm{XN},+\operatorname{mar}[70] / 46, \mathrm{XN}[30]$ (Figure 1-A1).

For Case 13, the sSMC derived from chromosome 18, with a karyotype of 47,XN,+mar (Figure 2-A1). However, CNV-seq results showed a triple-dosage increase of 18p11.32p11.21 (seq[hg19] 18p11.32p11.21(120000-14980000) x4) (Figure 2-A2).

G-banding analysis described the fetus' karyotype as $47, \mathrm{XN},+$ mar, without mosaicism, in Case 14 (Figure 2B1). There was a duplication of $9.26 \mathrm{Mb}$ at seq[hg19] 12p13.33p13.31(160000-9420000)x3 (spanning 100 OMIM genes) (Figure 2-B2). Thus, the sSMC originated from chromosome 12. For Case 15, traditional karyotype analysis showed $47, \mathrm{XN}$, +mar, accounting for $32 \%$ of all cultured amniocytes, and $68 \%$ of cultured cells showed 46,XN; that is, the karyotype was mosaicism 47,XN,+mar[26]/46,XN[74] (Figure 3A1). SNP array revealed a duplication of 8.9 Mb at arr[hg19]9p21.1p13.1 (30498773-39411673)X(2-3) in $40 \%$ of the fetal cells (Figure 3-A2). There were no abnormal ultrasonic manifestations throughout the whole pregnancy. For Case 16, standard karyotype analysis showed an abnormal karyotype of 47, XN, + mar (Figure 3-B1). The SNP array indicated a duplication of 1.8 Mb at arr[hg19]22q11.1q11.21(1687800218656495)X(4-5) in the fetal cells (Figure 3-B2).

\section{Pregnancy outcome and follow-up}

In our survey, among the 16 sSMC cases, contact was lost in 3 cases; 7 families chose termination of pregnancy $(7 / 16)$, whereas the pregnancy was continued in 6 cases $(6 / 16)$. The detailed information is shown in Table 1.

Specifically, the fetus of Case 1 was from a pedigree with hemophilia, and unfortunately, the fetus was diagnosed with hemophilia A by prenatal genetic diagnosis. The karyotype of Case 4 was $46, X,+m a r$, and the fetus would in all probability have Turner syndrome, even though the marker contained some genetic content. The fetus of Case 6 was detected by chromosomal analysis to involve Robertsonian translocation type trisomy 21. The couples in Cases 1, 4 and 6 terminated the pregnancy. Contact was lost in Cases 2, 3 
and 5. The sSMCs of Cases 8-11 were completely lacking euchromatin, and the babies were born normally and are at present 18 to 30 months old.

It is worth emphasizing that in Case 15 and Case 12, the babies were both healthy. The two children are currently approximately 2 (Case 12) or 3.5 years old (Case 15), with no clinical phenotypes observed. As the sSMCs of Cases 13,14 and 16 were all predicted to be pathogenic or of unknown significance, these pregnancies were terminated.

\section{Discussion}

In the current survey, we collected 16 SSMC prenatal samples, with an overall frequency of $0.0787 \%$, which is close to that of other research $[1,3]$. The origins of the sSMCs among our cases included acrocentric chromosomes (1/16), nonacrocentric chromosomes (4/16) and no $Y$ chromosomes, significantly different from other research by Leda Dalpra et al. and Bing Huang et al. $[13,14]$. This may be due to the small sample size of our study.

Reportedly, sSMCs deriving from acrocentric chromosomes with chromosome 15 are the most common type [15]; however, there was only one acrocentric chromosome with chromosome 22 in our study. This may be due to the limited number of SSMC cases we examined, and six of them lacked SNP or CNV-seq tests.

For case 7 to 11, the sSMCs contained negative genomic contents. The reason for the negative results was uncertain, but it may be due to sSMCs being derived from an acrocentric chromosome and containing only centromere heterochromatin without euchromatic regions.

For Case 12, the gain in the 9p12p21 region was evaluated as a variant of unknown significance. Supersonic ultrasound examination showed that the fetus was normal throughout the whole pregnancy. The baby grew normally and is at present a 2-year-old girl without any abnormalities. Therefore, we declare that the sSMC with a gain of approximately $10 \mathrm{Mb}$ of the 9p12p21 region is benign. For Case 13, the CNVseq result showed a triple-dosage increase of 18p11.32p11.21. This repetitive fragment encompasses 64 coding genes, of which 56 are OMIM genes, and there is insufficient evidence for triplosensitivity. Case ID [323729] is included in the DECIPHER database with a repetitive fragment slightly smaller than 18p11.32p11.21, and the main clinical symptom was central hypotonia. In Case ID [356973], the main clinical symptom was pathogenic. The DECIPHER database contains many cases that involve slightly larger repeats than the repeated fragment in case 14. The clinical manifestations included abnormal auricle, abnormal EEG, arched eyebrows, intellectual disability and multiple other malformations [394100] [394870] [394877] (likely pathogenic). The DGV database does not include reports of 18p11.32p11.21 duplication. Comprehensive analysis suggests that 18 p11.32p11.21 is likely pathogenic.

For Case 14, there was a duplication of $9.26 \mathrm{Mb}$ at the 12p13.33p13.31 region (spanning $100 \mathrm{OMIM}$ genes). Case ID [339519], with similar repeated fragments, is included in the DECIPHER database and likely pathogenic (de novo constitutive). For case ID [364080], clinical manifestations included EEG 
abnormalities, intellectual disability, prominent fingertip pads, ptosis, short nose, and type I diabetes mellitus. The DGV database does not include reports of 12p13.33p13.31 duplication. According to comprehensive analysis, the clinical significance of 12p13.33p13.31 gain was evaluated as a variant of unknown significance. However, the woman chose termination of pregnancyif the baby had any abnormality. We could not obtain definite clinical significance.

For Case 15, the karyotype was mosaicism 47,XN,+mar[32]/46,XN[68]. SNP array revealed a duplication of $8.9 \mathrm{Mb}$ in the $9 \mathrm{p} 21.1 \mathrm{p} 13.1$ region in $40 \%$ of the fetal cells, with the duplicated chromosomal section containing 68 OMIM genes, including ACO1, ALDH1B1 and APTX. In addition, there was insufficient evidence for triplosensitivity. The gain was evaluated as a variant of unknown significance. There were no abnormal ultrasonic manifestations throughout the whole pregnancy. The family continued the pregnancy, and the baby is now approximately 3.5 years old, with normal intellectual and physical development. Therefore, in our study, we could state that this gain is likely benign.

For Case 16, the SNP array detected a duplication of $1.8 \mathrm{Mb}$ at the 22q11.1q11.21 region in fetal cells. The duplicated chromosomal section, that is, the SSMC, contained the cat eye critical region (CECR) (Type I) on chromosome 22q11.21, which is definitely pathogenic.

The effect of sSMCs commonly depends on their origin, size, content, structure and proportion of mosaicism, as well as the amounts of euchromatin [16]. Others have reported that the risk of an abnormal phenotype may be associated with the size of the euchromatin region (even less than $1 \mathrm{Mb}$ ) or the number of genes (even less than 10). Nevertheless, when sSMCs contain some euchromatin fragments that are inherited from a healthy parent, we usually predict that they are likely benign[17]. As the morphology of sSMCs is highly heterogeneous, the genotype-phenotype correlation is exceedingly problematic in diagnostics.

Up to day ten SSMC-specific syndromes were described in literature [18-21]. In our study we could identify two of them: tetrasomy 18p syndrome (Case 13) and Cat eye syndrome(Case 16). Tetrasomy 18p is a rare disorder. The frequency of tetrasomy $18 p$ is $1 / 140,000$ in live newborns [22], which is the most common type among all these isochromosomes [23]. Their clinical characteristics include moderate to severe intellectual disability, developmental delay, microcephaly, typical dysmorphic features, and other anomalies, such as muscle tone abnormalities [24,25]. Cat eye syndrome (CES) is a rare developmental disorder with an incidence of $1 / 150,000$ to $1 / 50,000$ in liveborn infants[26]. CES occurs with a bisatellitedicentric SSMC(22), resulting in trisomy or partial tetrasomy of chromosome 22[27]. The clinical presentations of CES include high forehead, downslanting palpebral fissures, epicanthus, microphthalmia, cataract, and strabismus. Intellectual deficits, congenital heart defects and renal malformations may be involved in some severe cases [28-30].

It has been assumed that de novo SSMCs, particularly those with UPD, result from incomplete trisomy rescue[31]. However, to determine whether UPD coincides or is a consequence, further study should be performed. As chromosomes $6,7,14,15,16$, and 20 contain imprinting genes, UPDs of these chromosomes are most commonly reported in SSMC cases [32]. When sSMCs contain only 
heterochromatic regions, it is necessary to exclude whether an imprinted chromosome is involved in UPD [33]. This would be necessary due to the prognostic outcome, even though we have not found UPD in cases analyzed by SNP-array (cases 15 and 16). Other cases (7-14) in our study were analyzed by CNVseq which is not capable to detect UPD and this represent shortcoming in our research.Mosaic conditions were present in 5 cases (29.4\%), which was slightly lower than that reported in 2005 [13]. At the same time, the sSMC cell line level varied from $4 \%$ to $74 \%$, similar to previous literature [13].

Other researchers found that mosaicism is the reason for the normal or mild clinical manifestations in carriers of sSMCs with well-defined syndromes. No clinical manifestation is too expected in low-mosaic samples [20]. In our study, five of 16 cases involved mosaic sSMCs(Case 1, 3, 10,12 and 15). The children of Case 10,12 and 15 are with normal phenotypes up to now. The mosaicism level of Case 12 was $70 \%$, and the SSMC results in a normal phenotype. Similarly, in Case 16, duplication of the DNA sequence was approximately $8.9 \mathrm{Mb}$. Most likely involving mosaicism, the child is approximately 3.5 years old without any adverse clinical signs. Our results seemingly confirm the previous conclusion that mosaicism results in normal or minor clinical signs in carriers of sSMCs. Of course, long term development should be supervised, maybe the CNVs effects of these duplication regions has not been shown.

SNP array or CNV-seq enables describing the genetic content of SSMCs, making it easier for genetic counselors to establish genotype-phenotype correlations. Therefore, it is an outstanding technique and will be implemented in prenatal genetic diagnostics.

Notably, detailed molecular characterization of sSMCs might contribute to precise prenatal counseling. There are some limitations in the current study. First, consequent CNV-seq analyses of fetal tissues after termination of pregnancy were not conducted. Second, in Cases 8-11, CNV-seq analyses could not exclude the probability of UPD on the initial chromosome. Third, we could not obtain karyotyping using peripheral blood or other body tissues in Cases 12 and 15 .

In conclusion, our research emphasizes the combination of traditional cytogenetic and further molecular cytogenetic methods in the characterization of small marker chromosomes, which might facilitate a better understanding of the relationships between SSMCs and resulting phenotypes.

\section{Declarations}

\section{Acknowledgments:}

Not applicable

\section{Author contributions:}

$\mathrm{XK}$ and SH conceived of the study. SH summarized all the data and was involved in the histological analysis. $\mathrm{SH}$ and $\mathrm{XK}$ provided expertise for the data interpretation and suggestions for the manuscript preparation. SH wrote the manuscript. 


\section{Funding:}

This work was supported by grants from the Hospital Internal Funding from the First Affiliated Hospital of Zhengzhou University.

\section{Availability of data and material:}

All data generated or analyzed during this study are included in the article.

\section{Ethics approval and consent to participate:}

This study was approved by the Medical Ethics Committee of the First Affiliated Hospital of Zhengzhou University. All of the analyzed samples were obtained with signed informed consent.

\section{Consent for publication:}

All of the analyzed samples were obtained with signed informed consent.

\section{Competing interests:}

The authors declare that they have no competing interests.

\section{References}

1. Liehr T, Weise A. Frequency of small supernumerary marker chromosomes in prenatal, newborn, developmentally retarded and infertility diagnostics. Int J Mol Med. 2007; 19:719-731.

2. Santacroce R, Trunzo R, Leccese A, Pansini A, Gentile M, Margaglione M. The first case of a small supernumerary marker chromosome derived from chromosome 10 in an adult woman with an apparently normal phenotype. Syst Biol Reprod Med. 2015; 61:398-402.

3. Malvestiti F, De Toffol S, Grimi B, Chinetti S, Marcato L, Agrati C, et al. De novo small supernumerary marker chromosomes detected on 143,000 consecutive prenatal diagnoses: chromosomal distribution, frequencies, and characterization combining molecular cytogenetics approaches. Prenat Diagn. 2014; 34:460-468.

4. Jafari-Ghahfarokhi H, Moradi-Chaleshtori M, Liehr T, Hashemzadeh-Chaleshtori M, Teimori $H$, Ghasemi-Dehkordi P. Small supernumerary marker chromosomes and their correlation with specific syndromes. Adv Biomed Res. 2015; 4:140.

5. Stankiewicz P, Bocian E, Jakubow-Durska K, Obersztyn E, Lato E, Starke $\mathrm{H}$, et al. Identification of supernumerary marker chromosomes derived from chromosomes 5, 6, 19, and 20 using FISH. J Med Genet. 2000; 37:114-120.

6. Li T, Sang H, Chu G, Zhang Y, Qi M, Liu X, et al. Genotype-phenotype correlation in 75 patients with small supernumerary marker chromosomes. Mol Cytogenet. 2020; 13:30. 
7. Wu X, Xie X, Su L, Lin N, Liang B, Guo N, et al. Prenatal diagnosis of Pallister-Killian syndrome and literature review. J Cell Mol Med. 2021; 25:8929-8935.

8. Koc A, Onur SO, Ergun MA, Percin EF. Supernumerary marker chromosome 15 in a male with azoospermia and open bite deformity. Asian J Androl. 2009; 11:617-622.

9. Jaiswal SK, Kumar A, Ali A, Rai AK. Co-occurrence of mosaic supernumerary isochromosome 18p and intermittent 2q13 deletions in a child with multiple congenital anomalies. Gene. 2015; 559:94-98.

10. Chen CP, Ko TM, Chen YY, Su JW, Wang W. Prenatal diagnosis and molecular cytogenetic characterization of mosaicism for a small supernumerary marker chromosome derived from chromosome 22 associated with cat eye syndrome. Gene. 2013; 527:384-388.

11. Riggs ER, Andersen EF, Cherry AM, Kantarci S, Kearney H, Patel A, et al. Technical standards for the interpretation and reporting of constitutional copy-number variants: a joint consensus recommendation of the American College of Medical Genetics and Genomics (ACMG) and the Clinical Genome Resource (ClinGen). Genet Med. 2020; 22:245-257.

12. Riggs ER, Andersen EF, Cherry AM, Kantarci S, Kearney H, Patel A, et al. Correction: Technical standards for the interpretation and reporting of constitutional copy-number variants: a joint consensus recommendation of the American College of Medical Genetics and Genomics (ACMG) and the Clinical Genome Resource (ClinGen). Genet Med. 2021.

13. Dalpra L, Giardino D, Finelli P, Corti C, Valtorta C, Guerneri S, et al. Cytogenetic and molecular evaluation of 241 small supernumerary marker chromosomes: cooperative study of 19 Italian laboratories. Genet Med. 2005; 7:620-625.

14. Huang B, Solomon S, Thangavelu M, Peters K, Bhatt S. Supernumerary marker chromosomes detected in 100,000 prenatal diagnoses: molecular cytogenetic studies and clinical significance. Prenat Diagn. 2006; 26:1142-1150.

15. Crolla JA, Youings SA, Ennis S, Jacobs PA. Supernumerary marker chromosomes in man: parental origin, mosaicism and maternal age revisited. Eur J Hum Genet. 2005; 13:154-160.

16. Guediche N, Tosca L, Nouchy M, Lecerf L, Cornet D, Brisset S, et al. Small supernumerary marker chromosomes derived from chromosomes 6 and 20 in a woman with recurrent spontaneous abortions. Eur J Med Genet. 2012; 55:737-742.

17. Liehr T, Ewers E, Kosyakova N, Klaschka V, Rietz F, Wagner R, et al. Handling small supernumerary marker chromosomes in prenatal diagnostics. Expert Rev Mol Diagn. 2009; 9:317-324.

18. Chen J, Guo M, Luo M, Deng S, Tian Q. Clinical characteristics and management of Turner patients with a small supernumerary marker chromosome. Gynecol Endocrinol. 2021; 37:730-734.

19. Jang W, Chae H, Kim J, Son JO, Kim SC, Koo BK, et al. Identification of small marker chromosomes using microarray comparative genomic hybridization and multicolor fluorescent in situ hybridization. Mol Cytogenet. 2016; 9:61.

20. Liehr T, Al-Rikabi A. Mosaicism: Reason for Normal Phenotypes in Carriers of Small Supernumerary Marker Chromosomes With Known Adverse Outcome. A Systematic Review. Front Genet. 2019; 10:1131. 
21. Sheridan MB, Kato T, Haldeman-Englert C, Jalali GR, Milunsky JM, Zou Y, et al. A palindrome-mediated recurrent translocation with 3:1 meiotic nondisjunction: the t(8;22)(q24.13;q11.21). Am J Hum Genet. 2010; 87:209-218.

22. Schwemmle C, Arslan-Kirchner M, Pabst B, Ptok M. [Tetrasomy 18p syndrome and hearing loss. An unusual case]. HNO. 2012; 60:901-905.

23. Kotzot D, Bundscherer G, Bernasconi F, Brecevic L, Lurie IW, Basaran S, et al. Isochromosome 18p results from maternal meiosis II nondisjunction. Eur J Hum Genet. 1996; 4:168-174.

24. Wei J, Xie Y, He W, Liu W, Jian W, Chen M, et al. Clinical outcome: a monosomy 18p is better than a tetrasomy 18p. Cytogenet Genome Res. 2014; 144:294-298.

25. Bawazeer S, Alshalan M, Alkhaldi A, AlAtwi N, AlBalwi M, Alswaid A, et al. Tetrasomy 18p: case report and review of literature. Appl Clin Genet. 2018; 11:9-14.

26. Hernandez-Medrano C, Hidalgo-Bravo A, Villanueva-Mendoza C, Bautista-Tirado T, Apam-Garduno D. Mosaic cat eye syndrome in a child with unilateral iris coloboma. Ophthalmic Genet. 2021; 42:84-87.

27. Belien V, Gerard-Blanluet M, Serero S, Le Du N, Baumann C, Jacquemont ML, et al. Partial trisomy of chromosome 22 resulting from a supernumerary marker chromosome 22 in a child with features of cat eye syndrome. Am J Med Genet A. 2008; 146A:1871-1874.

28. AlSubaihin A, VanderMeulen J, Harris K, Duck J, McCready E. Mullerian Agenesis in Cat Eye Syndrome and 22q11 Chromosome Abnormalities: A Case Report and Literature Review. J Pediatr Adolesc Gynecol. 2018; 31:158-161.

29. Melo C, Gama-de-Sousa S, Almeida F, Rendeiro P, Tavares P, Cardoso H, et al. Cat eye syndrome and growth hormone deficiency with pituitary anomalies: a case report and review of the literature. Gene. 2013; 529:186-189.

30. Alamer L, Bassant S, Alhazmi R, Alzahrani M. Rare otologic presentation of cat eye syndrome. Ann Saudi Med. 2019; 39:441-443.

31. Matsubara K, Yanagida K, Nagai T, Kagami M, Fukami M. De Novo Small Supernumerary Marker Chromosomes Arising From Partial Trisomy Rescue. Front Genet. 2020; 11:132.

32. Zucker K, Worcel A. The histone H3/H4.N1 complex supplemented with histone H2A-H2B dimers and DNA topoisomerase I forms nucleosomes on circular DNA under physiological conditions. J Biol Chem. 1990; 265:14487-14496.

33. Liehr T, Ewers E, Hamid AB, Kosyakova N, Voigt M, Weise A, et al. Small supernumerary marker chromosomes and uniparental disomy have a story to tell. $J$ Histochem Cytochem. 2011; 59:842-848.

\section{Tables}

Table 1. Summary of Karyotypes, CNV-seq or SNP-array results and follow-ups 


\begin{tabular}{|c|c|c|c|c|}
\hline NO & Karyotypes & CNV-seq or SNP-array & Significance & Follow-ups \\
\hline 1 & $47, \mathrm{XN},+\operatorname{mar}[74] / 46, \mathrm{XN}[26]$ & N.A & N.A & abort \\
\hline 2 & $47, \mathrm{XN},+\mathrm{mar}$ & N.A & N.A & lose contact \\
\hline 3 & $47, \mathrm{XN},+\operatorname{mar}[8] / 46, \mathrm{XN}[92]$ & N.A & N.A & lose contact \\
\hline 4 & $46, \mathrm{X},+\mathrm{mar}$ & N.A & N.A & abort \\
\hline 5 & $47, \mathrm{XN},+\mathrm{mar}$ & N.A & N.A & lose contact \\
\hline 6 & $\begin{array}{l}47, \mathrm{XN}, \mathrm{rob}(21 ; 21) \\
\text { (q10;q10),+21,+mar }\end{array}$ & N.A & N.A & abort \\
\hline 7 & $46, \mathrm{X},+\mathrm{mar}$ & seq[hg19]45,XO & negative & abort \\
\hline 8 & $47, \mathrm{XN},+\mathrm{mar}$ & seq[hg19] 46,XN & negative & continue \\
\hline 9 & $47, \mathrm{XN},+\mathrm{mar}$ & seq[hg19] 46,XN & negative & continue \\
\hline 10 & $\begin{array}{l}47, X N,+m a r[4] / 46 \otimes \\
\text { XN[96] }\end{array}$ & seq[hg19] 46,XN & negative & continue \\
\hline 11 & $47, \mathrm{XN},+\mathrm{mar}$ & seq[hg19] 46,XN & negative & continue \\
\hline 12 & $\begin{array}{l}47, \mathrm{XN},+\operatorname{mar}[70] / 46 \mathrm{X} \\
\mathrm{XN}[30]\end{array}$ & $\begin{array}{l}\text { seq[hg19] 9p12p21(28600000- } \\
38780000) \times 3 \varangle 40 \% \rrbracket\end{array}$ & $\begin{array}{l}\text { variant of } \\
\text { unknown } \\
\text { significance }\end{array}$ & $\begin{array}{l}\text { continue, } 2 \\
\text { years old }\end{array}$ \\
\hline 13 & $47, \mathrm{XN},+\mathrm{mar}$ & $\begin{array}{l}\text { seq[hg19] } \\
\text { 18p11.32p11.21(120000- } \\
\text { 14980000) x4 }\end{array}$ & $\begin{array}{l}\text { likely } \\
\text { pathogenic }\end{array}$ & abort \\
\hline 14 & $47, \mathrm{XN},+\mathrm{mar}$ & $\begin{array}{l}\text { seq[hg19] } \\
\text { 12p13.33p13.31(160000- } \\
9420000) \times 3\end{array}$ & $\begin{array}{l}\text { variant of } \\
\text { unknown } \\
\text { significance }\end{array}$ & abort \\
\hline 15 & $\begin{array}{l}47, \mathrm{XN},+\operatorname{mar}[26] / 46 \\
\mathrm{XN}[74]\end{array}$ & $\begin{array}{l}\text { arr[hg19]9p21.1p13.1(30498773- } \\
39411673) \times(2-3)\end{array}$ & $\begin{array}{l}\text { likely } \\
\text { benign }\end{array}$ & $\begin{array}{l}\text { continue,3.5 } \\
\text { years old }\end{array}$ \\
\hline 16 & $47 \rrbracket \mathrm{XN},+\mathrm{mar}$ & $\begin{array}{l}\text { arr[hg19]22q11.1q11.21(16878002- } \\
18656495) \times(4-5)\end{array}$ & pathogenic & abort \\
\hline
\end{tabular}

\section{Figures}


A1

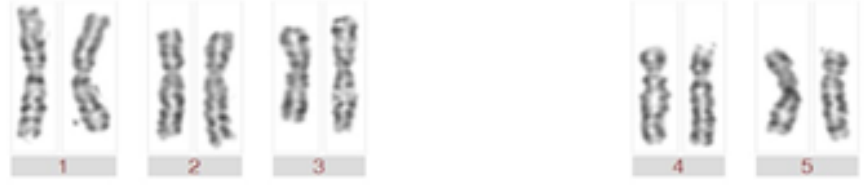

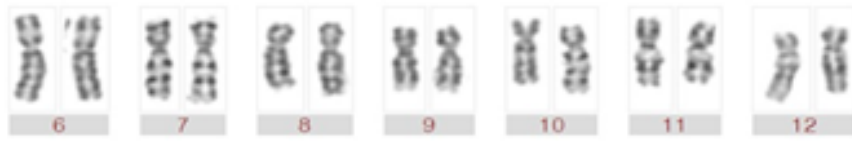

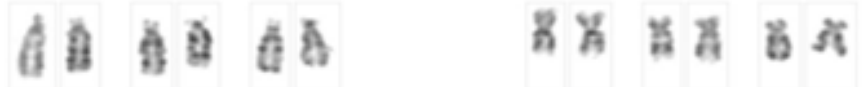

\begin{tabular}{|l|l|l|l|l|l|}
\hline 13 & 14 & 15 & 16 & 18 \\
\hline
\end{tabular}

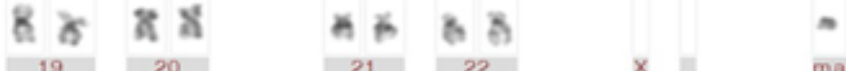

A2

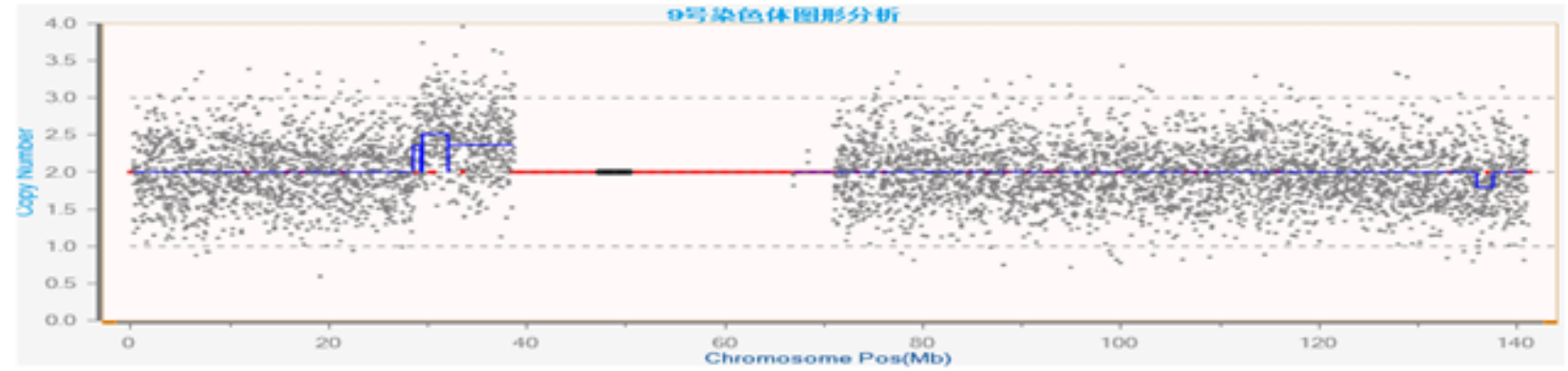

Figure 1

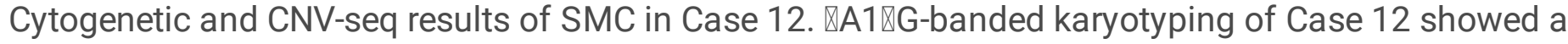

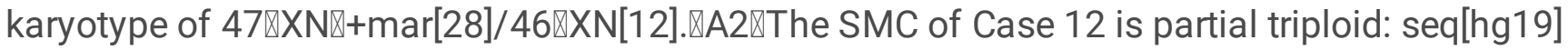
$9 \mathrm{p} 12 \mathrm{p} 21(28600000-38780000) \times 3(40 \%)$. 


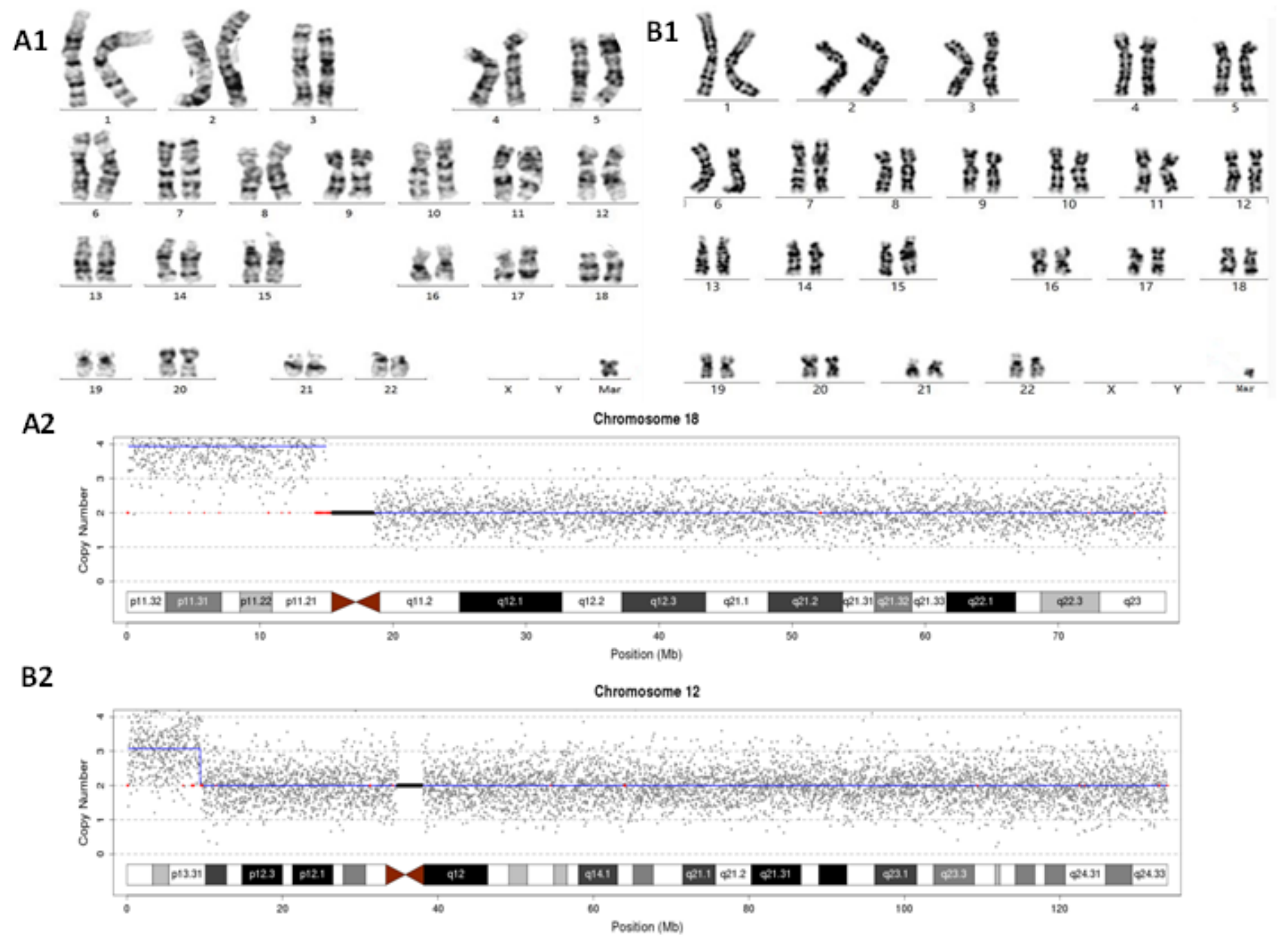

Figure 2

Cytogenetic and CNV-seq results of SMCs in Cases 13 and 14. (A1) G-banded karyotyping of case 13 showed a karyotype of $47, \mathrm{XN},+$ mar. (A2) The SMC of Case 13 is a partial tetraploid: seq[hg19] 18p11.32p11.21(120000-14980000) x4 (B1) G-banded karyotyping of Case 14 showed a karyotype of 47, $\mathrm{XN},+$ mar. (B2) The SMC in Case 14 is a partial triploid: seq[hg19] 12p13.33p13.31(160000-9420000)x3. 
A1

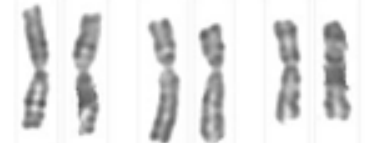

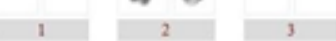

ใด้

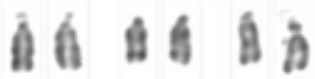

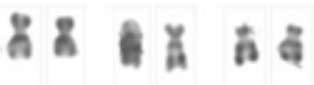

13 14 is

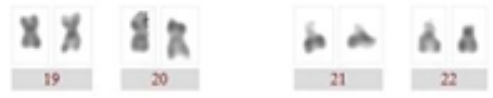

B1
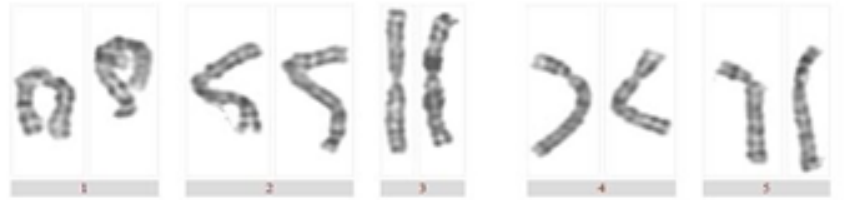

c( 581118

IIII

वे $x$ क

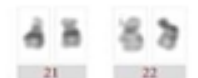

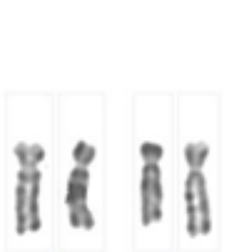

A2

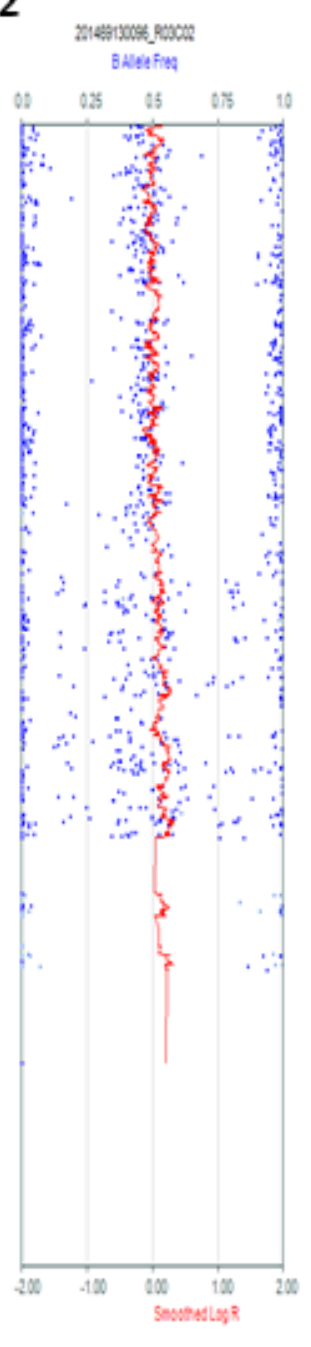

B2

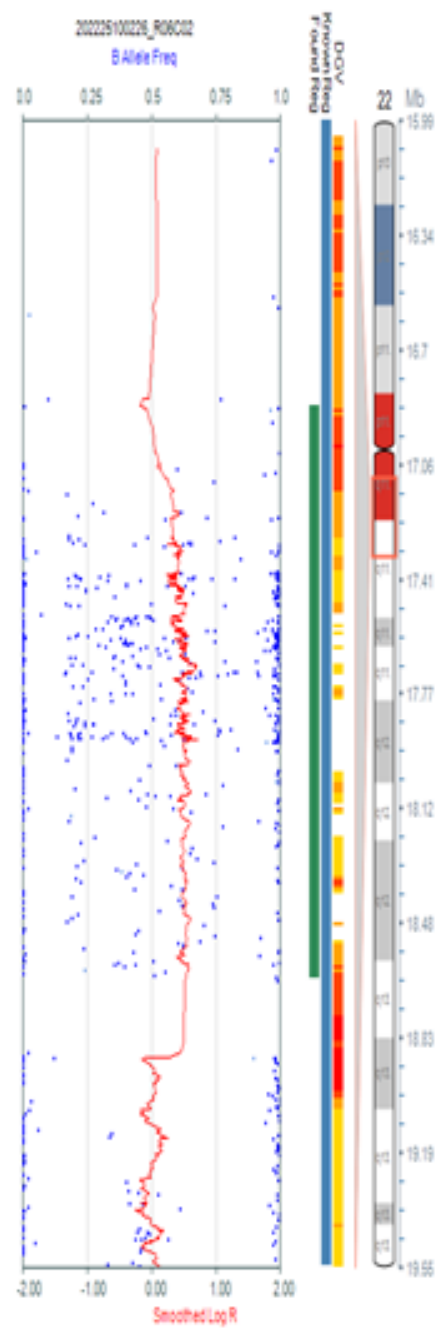

\section{Figure 3}

Cytogenetic and SNP array results of SMCs in Cases 15 and 16. (A1) A1 G-banded karyotyping of Case 15 showed a karyotype of $47, \mathrm{XN},+\operatorname{mar}[16] / 46, \mathrm{XN}[44]$. (A2) The SMC of case 15 is a partial triploid: arr[hg19]9p21.1p13.1(30498773-39411673)X(2 3). (B1) G-banded karyotyping of Case 16 showed a karyotype of $47, \mathrm{XN},+$ mar. (B2) The SMC of Case 16 is a partial tetraploid or pentaploid: arr[hg19]22q11.1q11.21(16878002-18656495)×(4 5). 